\title{
Imaging nano-scale structure ordering by measuring phase field mapping using holographic reconstruction
}

Jing Tao and J. M. Zuo

Department of Materials Science and Engineering and Materials Research Laboratory, University of Illinois at Urbana and Champaign, 1304 West Green Street, Urbana Illinois 61801

We report a quantitative technique that can be used to measure nanometer-sized structural ordering by mapping the phase using holographic reconstruction. We apply this technique to study the important problem of charge ordering in manganese oxides which have many applications in spintronics and magnetic sensor.

Doped $\mathrm{La}_{1-\mathrm{x}} \mathrm{Ca}_{\mathrm{x}} \mathrm{MnO}_{3}(0.5<\mathrm{x}<0.8)$ has complex temperature-dependent nanoscale structural features during the charge ordering transition. It has been studied by many different experimental techniques in the past several years [1-2], but the nature of charge ordering remains unknown. To quantify the temperature-dependent structure evolution, we study the phase transition by in-situ electron nanodiffraction and dark field lattice imaging of $\mathrm{La}_{0.33} \mathrm{Ca}_{0.67} \mathrm{MnO}_{3}$ at temperatures from $100 \mathrm{~K}-300 \mathrm{~K}$.

$\mathrm{La}_{0.33} \mathrm{Ca}_{0.67} \mathrm{MnO}_{3}$ has a high-temperature cubic and room temperature distorted-perovskite structure $\left(\mathrm{a} \approx \mathrm{c}, \mathrm{b}=\sqrt{2} \mathrm{a}_{\mathrm{p}}\right)$. At low temperature the ordered structure has a supercell along a-axis 3 times the orthorhombic cell. Electron diffraction pattern from single crystal domain of $\mathrm{La}_{0.33} \mathrm{Ca}_{0.67} \mathrm{MnO}_{3}$ specimen at [010] zone axis show the superlattice reflections pointing out by arrows (Fig. 1a)). Quantitative analysis of the electron diffraction data shows a continuous commensurateincommensurate charge ordering transition during the melting process of charge ordering (Fig. 1b)). In real space, we observe this charge ordering structure as "stripes" using dark field imaging (Fig. 1c)) at low temperatures. Using the superlattice reflections satellite on (202) reflection in the twobeam diffraction condition shown as Fig. 1d), the intensity contrast of the image is directly from the charge ordering superstructure modulation. Charge ordering defects are clearly shown in these images. To map the distribution of defects, we extract the phase from the images by using holographic reconstruction. Fig. 2a)-d) show the schematic reconstruction process. A phase jump is used to describe a charge ordering defect between periodic commensurate stripes. We compare the evolution of the phase field with the electric property measurement and find a correlation between the rise of resistivity and the decreasing of defects density (Fig. 2e)) upon cooling. The results here, together with our previous electron microdiffraction study in $\mathrm{La}_{1-\mathrm{x}} \mathrm{Ca}_{\mathrm{x}} \mathrm{MnO}_{3}$ with $\mathrm{x}=1 / 3$ [3], shows the importance of charge ordering to the unusual properties of this material [4].

\section{Reference}

[1] C. H. Chen et al., J. Appl. Phys. 81, 4326 (1997)

[2] P. G. Radaelli et al., Phys. Rev. B 59, 14440 (1999)

[3] J. M. Zuo and J. Tao, Phys. Rev. B $63060407(2000)$

[4] This work is supported by DOE DEFG02-01ER45923 and DEFG02-91ER45439. The experiments were carried at the CMM, MRL, UIUC. We thank Dr. R. Twesten for the help with JEOL2010F electron microscope. 


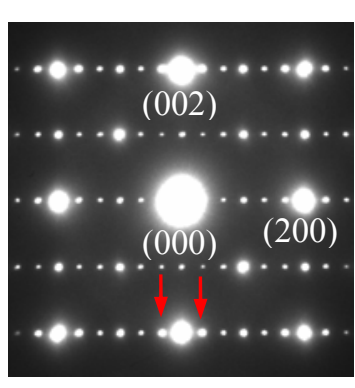

a)

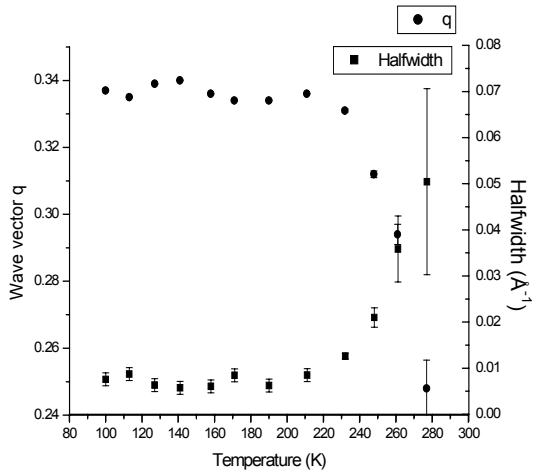

b)

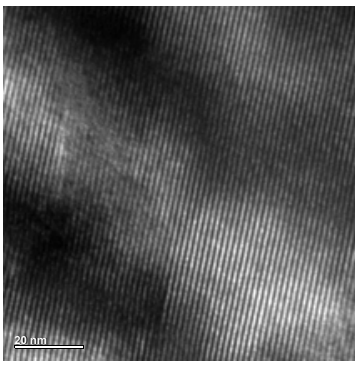

c)

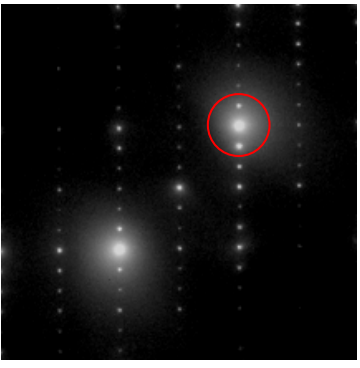

d)

Figure 1. a) Electron diffraction patterns at [010] zone axis of $\mathrm{La}_{0.33} \mathrm{Ca}_{0.67} \mathrm{MnO}_{3}$ at $\mathrm{T}=100 \mathrm{~K}$. b) Wave vector q (circle dots) and halfwidth (square dots) versus temperature. A dark field image c) shows the stripe-like structure with defects for $\mathrm{La}_{0.33} \mathrm{Ca}_{0.67} \mathrm{MnO}_{3}$ at $\mathrm{T}=98 \mathrm{~K}$. The image is taken under the diffraction condition d) with an aperture including (202) Bragg peak and the superlattice peaks around it shown inside the red circle.
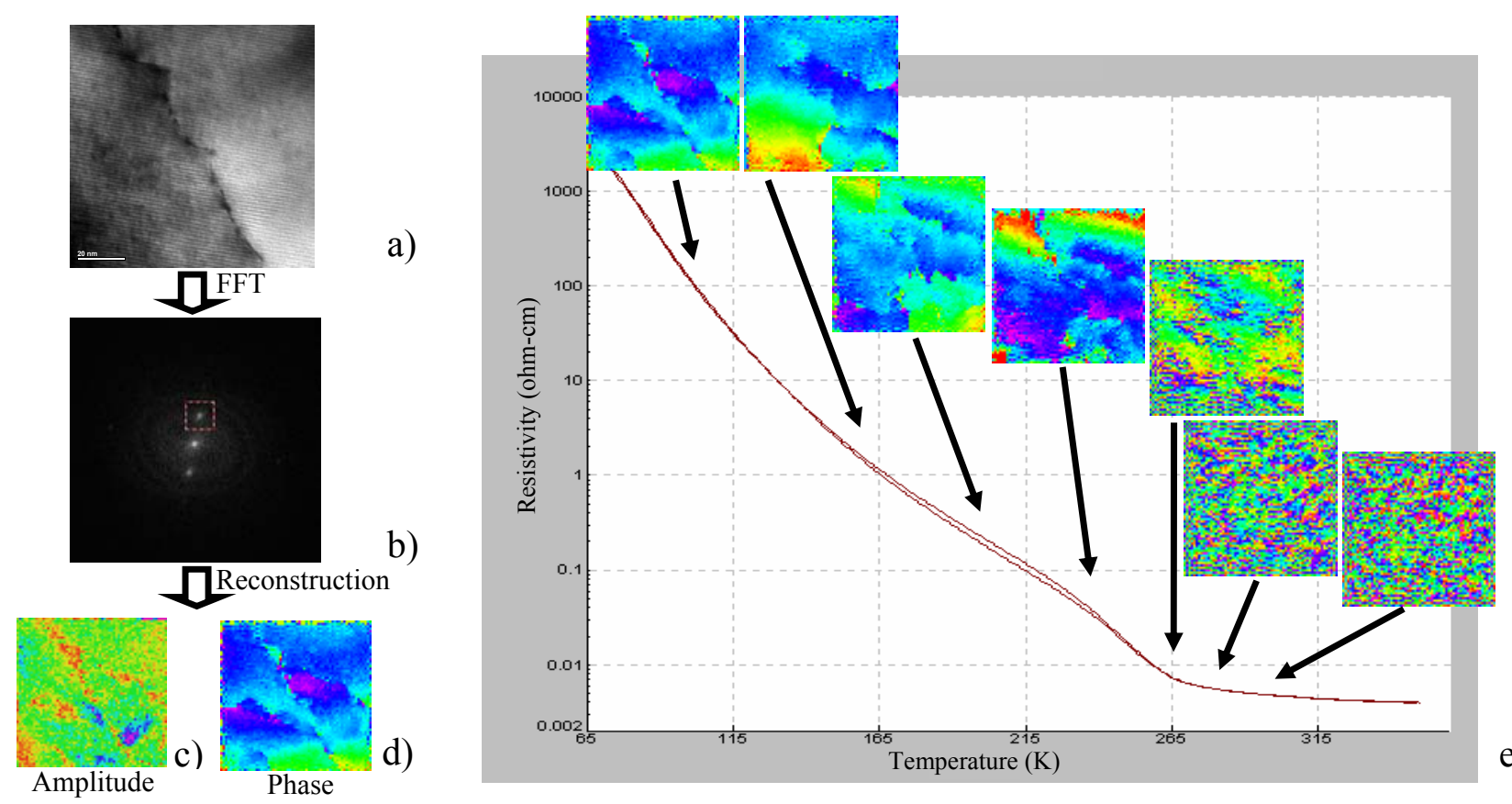

e)

Figure 2. The left part is a schematic representation of the reconstruction process. a) A DF real space image with a crystal defect goes through it. b) The Fourier transformation of image a). There are three bands shown on b). We selected one of the side bands inside the dashed square area, cut it out and reconstructed the amplitude field c) and phase field $d$ ). Phase field evolution combined with the resistivity measurement is shown on right e). All the phase fields are reconstructed from real space images on a fixed area of $\mathrm{La}_{0.33} \mathrm{Ca}_{0.67} \mathrm{MnO}_{3}$ specimen taken with decreasing temperature at $\mathrm{T}=295 \mathrm{~K}$, $280 \mathrm{~K}, 265 \mathrm{~K}, 235 \mathrm{~K}, 200 \mathrm{~K}, 160 \mathrm{~K}$ and $98 \mathrm{~K}$. 